\title{
Distinct helical molecular orbitals through conformational lock
}

Ani Ozcelik, ${ }^{\text {ta }}$ Daniel Aranda, ${ }^{\text {tb,c }}$ Sara Gil-Guerrero, ${ }^{d}$ Xaquín A. Pola-Otero, ${ }^{a}$ Maria Talavera, ${ }^{e}$ Liangxuan Wang, ${ }^{f}$ Santosh Kumar Behera, ${ }^{f}$ Johannes Gierschner, ${ }^{f}$ Ángeles Peña-Gallego, ${ }^{d}$ Fabrizio Santoro, ${ }^{* b}$ Raquel Pereira-Cameselle, ${ }^{* a}$ and J. Lorenzo Alonso-Gómez*a

\footnotetext{
a.Departamento de Química Orgánica, Universidade de Vigo, Lagoas-Marcosende, Vigo 36310, Spain, e-mail: raquel@uvigo.es, lorenzo@uvigo.es

b. Istituto di Chimica dei Composti Organometallici, Consiglio Nazionale delle Ricerche, Pisa, Italy, e-mail: fabrizio.santoro@pi.iccom.cnr.it

c. Departamento de Química Física, Universidad de Málaga, Bulevar Louis Pasteur 31, Málaga 29010, Spain

d. Departamento de Química Física, Universidade de Vigo, Lagoas-Marcosende, Vigo 36310, Spain

e. Department of Chemistry, Humboldt Universität zu Berlin, Brook-Taylor-Strasse 2, 12489 Berlin, Germany

f. Madrid Institute for Advanced Studies, IMDEA Nanoscience, C/ Faraday 9, Ciudad Universitaria de Cantoblanco, 28049 Madrid, Spain + These authors contributed equally to this work.
}

Several theoretical studies have proposed strategies to reach helical molecular orbitals (HelMOs) in [ $n]$ cumulenes. While chiral even- $[n]$ cumulenes feature Hel-MOs, odd- $[n]$ cumulenes may also present them if the terminal groups lie on different planes. However, the hitherto proposed systems have been either experimentally unfeasible or resulted in opposite pseudodegenerated Hel-MOs, impeding their use in real applicatons. To overcome this challenge, we hereby demonstrate the introduction of a remarkable energy difference between helical orbitals of opposite twist by fixing the torsion angle between the terminal groups in butadiyne fragments. In order to experimentally lock the conformation of the terminal groups, we designed cyclic architectures by combining acetylenes with chiral spirobifluorenes. A straightforward synthetic strategy along with the high stability allowed the isolation and full characterization of systems presenting distinct helical orbitals. Finally, a thorough computational analysis revealed that the most significant optical responses of these systems originate mainly from the exciton coupling between the featured diphenylbutadiyne fragments. This novel strategy opens now access to the development of systems with distinct helical molecular orbitals suitable for their implementation into chiroptical and optoelectronic applications

\section{Introduction}

Linear carbon moieties serve as versatile building blocks for the exploration of linear, cyclic and cage-like systems with diverse topologies. Acetylene, the shortest of the series with two sp-hybridised carbon atoms, has been extensively used to linearly connect different or identical moieties. ${ }^{1}$ Allene is the next with an sphybridised central carbon connected to two $\mathrm{sp}^{2}$ carbons through double bonds. ${ }^{2}$ Such species incorporating consecutive double bonds are often referred to as $[n]$ cumulenes, where $[n]$ is the number of consecutive double-bonds. ${ }^{3}$ Within this family, the end-groups are placed in the same plane when $[n]$ is odd, or in orthogonal planes when $[n]$ is even (Scheme 1). In the latter case, axial chirality is the outcome if two different substituents are present at each end. Therefore, allenes have been widely employed for the construction of diverse morphologies. ${ }^{4}$

Theoretical simulations have shown that even-[ $n]$ cumulenes exhibit helical molecular orbitals (Hel-MOs) connecting the two $p$ orbitals of the terminal carbon atoms (Scheme 1). ${ }^{5}$ However, since the two possible paths through a left or right twist about the cumulene bonds are equivalent, two degenerated molecular

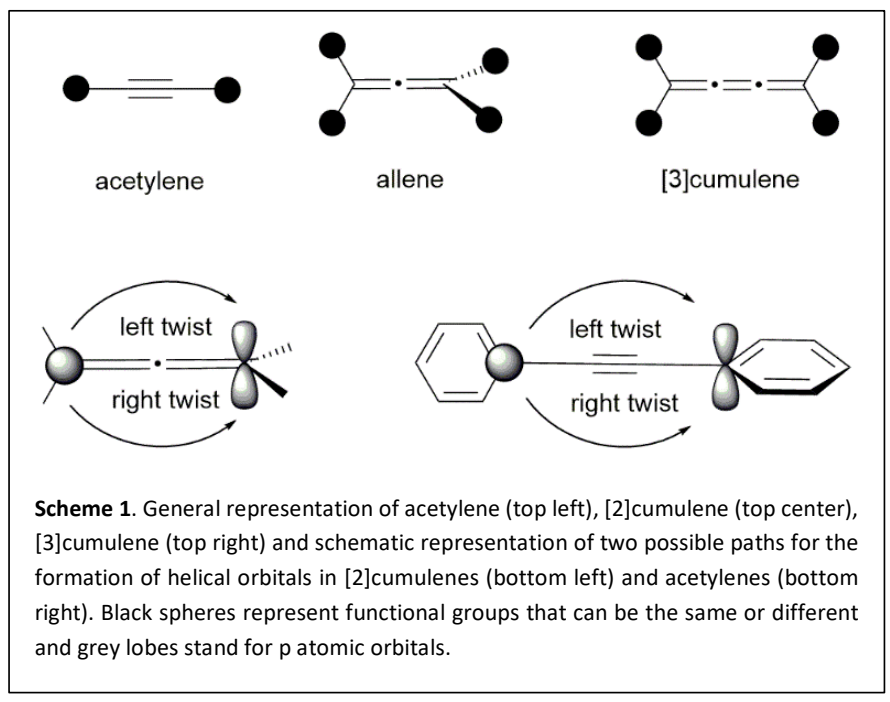


orbitals (MOs) with opposite helicities arise. In search of the real applicability of these systems, further studies have sought an energy separation between the orbitals of opposite helicities by the incorporation of certain peripheral substituents. ${ }^{6}$

Hel-MOs have also been predicted for odd- $[n]$ cumulenes, in which the two terminal $\mathrm{sp}^{2}$ hybridised carbons are forced to be in different planes. ${ }^{7}$ Nevertheless, experimental analysis of such analogues is probably hampered by the instability of cumulenes. On the other hand, in acetylene-bearing molecules, practicallydegenerated helical orbitals have been predicted if the torsion angles between the terminal groups are close to $90^{\circ} .5,8$ However, even when a particular conformation may present non-degenerated helical orbitals, the existence of the corresponding enantiomeric conformation provides degenerated opposite Hel-MOs. ${ }^{9}$

The recent theoretical demonstration of Solomon and co-workers on the presence of circular currents in molecules featuring Hel-MOs calls for the development of real systems with Hel-MOS for spintronics. ${ }^{10}$ This emerging technology, which controls the spin of electrons, presents higher energy efficiency and frequency as compared to electronics. ${ }^{11}$ In this regard, chiral organic molecules manifesting chiral-induced spin selectivity (CISS) ${ }^{12}$ have already shown great advantages in memory devices. ${ }^{13,14}$ Additionally, CISS has been successfully exploited in relevant fields such as enantiomeric resolution ${ }^{15}$ or catalysis. ${ }^{16}$ Thus, the development of robust systems with Hel-MOs would significantly boost these fields. Herein, we present a straightforward strategy to construct systems featuring distinct helical MOs by controlling the torsion angle between the terminal groups of oligoynes in cyclic structures. We start by demonstrating that the control of the torsion angle between the phenyl rings in diphenylbutadiyne (DPBD) can be employed to generate helical MOs. Based on this model, we designed cyclic acetylenic spirobifluorenes (SBFs) in order to lock the torsion angle between the terminal groups of the butadiyne moieties. Thereafter, the efficient synthesis and full characterization enabled the unambiguous structural determination including the absolute configuration. Finally, theoretical analysis uncovers that the different DPBD moieties synchronize according to the exciton coupling theory ${ }^{17}$ and exhibit Hel-MOs.

\section{Results and discussion}

\section{Theoretical model}

In order to understand the nature of Hel-MOs in oligoynes, we calculated the MOs of DPBD at different torsion angles between the two aromatic rings by density functional theory (DFT) at the B3LYP/6-31G(d,p) level of theory as implemented in Gaussian 09. ${ }^{18}$ Among all MOs, our attention was drawn to HOMO-4 and HOMO-5 since these are the occupied MOs with the most remarkable helical character (Figure 1 center, for more details see the supporting information (SI)).

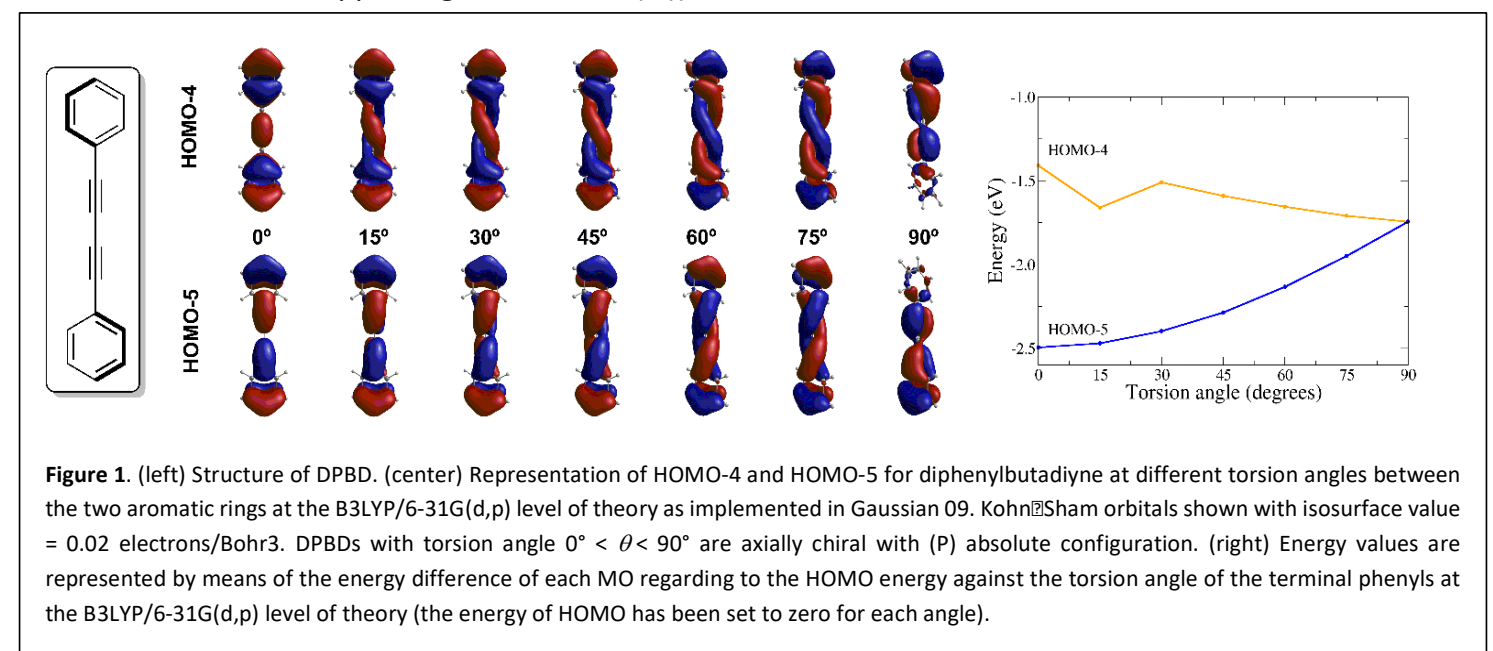

We noticed that the torsion angle does not only dictate the energy, but also it dramatically affects the helical character of the MOs. Figure 1 shows these MOs for torsion angles of $0^{\circ}, 15^{\circ}, 30^{\circ}, 45^{\circ}, 60^{\circ}, 75^{\circ}$ and $90^{\circ}$. As earlier demonstrated for odd-[ $n]$ cumulenes, ${ }^{19}$ there are no Hel-MOs if the symmetry is $D_{2 h}$, i.e., the torsion angle is $0^{\circ}$. Two enantiomeric degenerated Hel-MOs appear when the angle between terminal sp $\mathrm{s}^{2}$ carbons is $90^{\circ}$. Yet, while HOMO-4 presents a clear $(M)$ helicity for the angles between $15^{\circ}$ and $75^{\circ}, \mathrm{HOMO}-5$ is of opposite helicity, $(P)$, and only for angles between $45^{\circ}$ and $75^{\circ}$. Accordingly, structures presenting torsion angles in the range of $15^{\circ}$ to $30^{\circ}$ manifest only one helicity in the pseudo-frontier occupied MOs. Additionally, 
the energy difference between HOMO-4 and HOMO-5 for torsion angles within this range is about $1 \mathrm{eV}$ (Figure 1 right). Based on this model, we foresee that systems incorporating DPBD fragments with fixed torsion angles varying from $15^{\circ}$ to $30^{\circ}$ will overcome the challenge of response cancellation in state-of-theart systems due to the presence of degenerate or pseudo-degenerate orbitals featuring opposite helicities. Therefore, exploring the impact arising from Hel-MOs in real systems should be now accessible with this strategy.

\section{Design of conformationally locked oligoynes}

We have earlier demonstrated the feasibility of chiral axes for the construction of cyclic ${ }^{20}$ and cage-like ${ }^{21}$ structures with well-defined geometries. However, the use of allenes for real applications is limited by isomerization under daylight, ${ }^{22}$ or even in the dark when attached to electron-rich substituents. ${ }^{23}$ On the other hand, spirobifluorenes (SBFs) have been employed as building blocks in molecular electronics for the last two decades due to their high stability. ${ }^{24}$ Since such compounds may also feature axial chirality if adequately functionalised, we theoretically proposed SBFs as a robust alternative to allenes. ${ }^{25}$

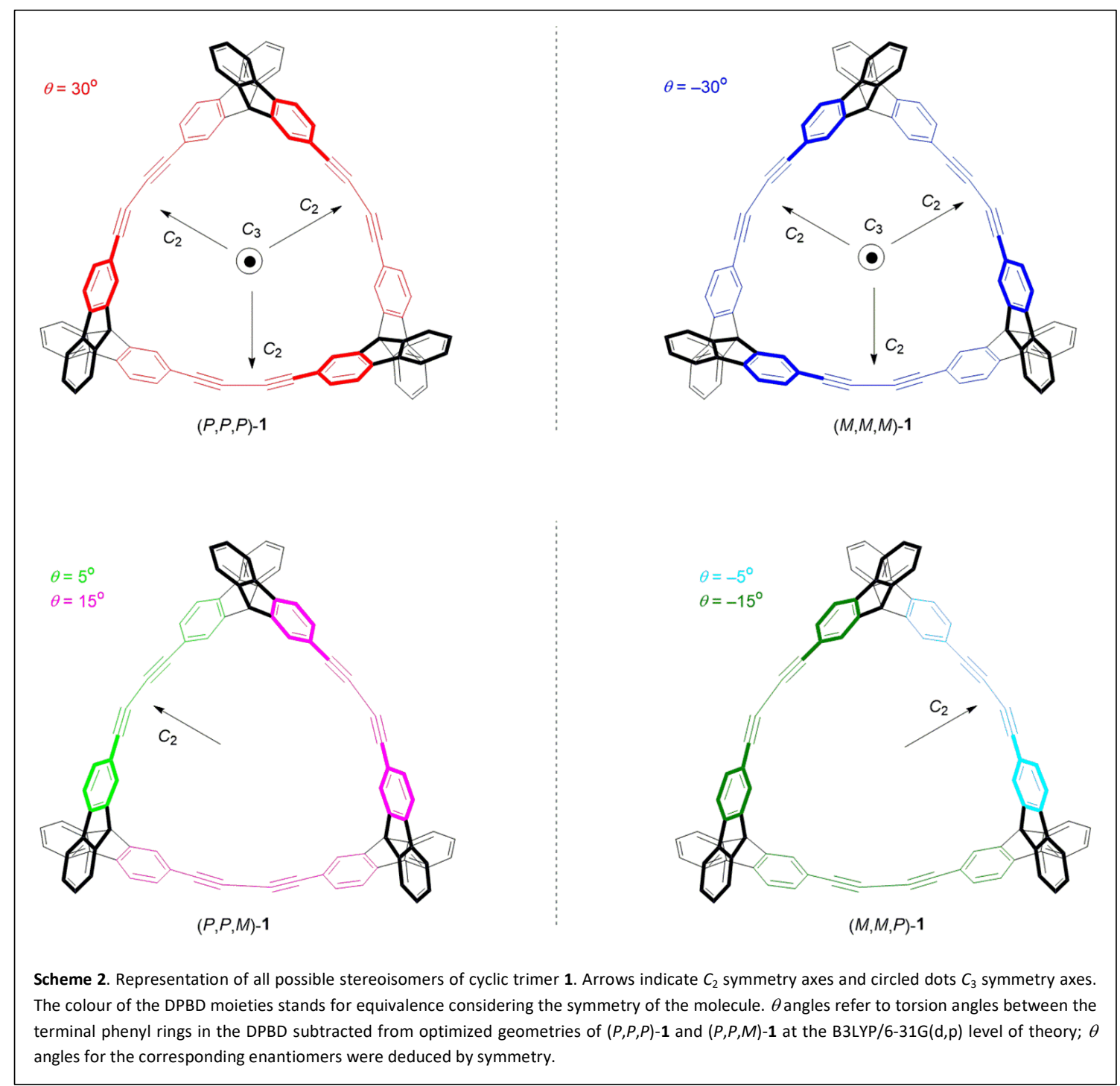

More recently, our synthetic methodologies have opened access to surface functionalization with SBFs using thioacetates, ${ }^{26}$ as well as tuning of the optical properties by the incorporation of metallaaromatic rings. ${ }^{27}$ In addition, we performed the cyclic oligomerization of diethynylspirobifluorene (DES) leading to all-carbon double helices and flexible shape-persistent macrocycles. ${ }^{28}$ Whereas both systems involve two and four 
DPBD moieties, respectively, the torsion angle between the phenyl groups is ca. $90^{\circ}$ in the former and very broad in the latter, and hence not adequate for Hel-MOs. To fix the torsion angle in the above-deduced optimal range of $15^{\circ}-30^{\circ}$, we designed cyclic systems bearing three axially chiral DES units. Regarding the stereochemistry of these cyclic trimers, there are four possible stereoisomers: $(P, P, P)-\mathbf{1},(M, M, M)-\mathbf{1},(P, P, M)$ 1 and $(M, M, P)-\mathbf{1}$ (Scheme 2).Geometry optimization at the B3LYP/6-31G(d,p) level of theory of diastereoisomers $(P, P, P)-\mathbf{1}$ and $(P, P, M)$-1 revealed structures with $D_{3}$ and $C_{2}$ symmetry, respectively (for more information, see the $\mathrm{SI})$. While homochiral $(P, P, P)-\mathbf{1}$ has three equivalent DPBD fragments with torsion angle $\theta=30^{\circ}$ between the terminal phenyl rings, heterochiral $(P, P, M)-1$ is composed of two equivalent DPBD with $\theta=15^{\circ}$ and the other with $\theta=5^{\circ}$. The corresponding enantiomers exhibit opposite torsion angles. Since all stereoisomers of 1 feature at least one DPBD with $\theta$ absolute value of $15^{\circ}-30^{\circ}$, all were considered of interest for the exploration of Hel-MOs.

\section{Synthesis of cyclic oligoynes}

The synthesis of the cyclic oligoynes was carried out through Sonogashira cross-coupling reaction of commercially available racemic dibromo-SBF ( $\quad$ )-2 with trimethylsilylacetylene and subsequent trimethylsilyl deprotection to give $( \pm)-3$ in $75 \%$ yield over two steps (Scheme 3, for experimental details see the $\mathrm{SI}$ ). Cyclooligomerization reactions of

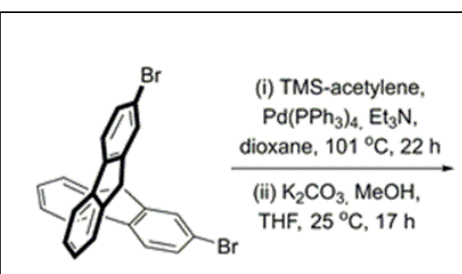

$( \pm)-2$

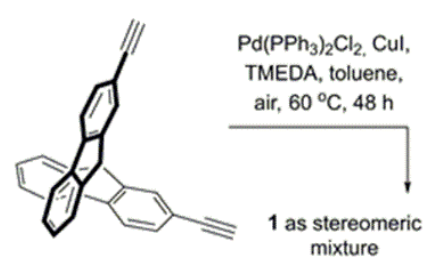

( \pm -3

Scheme 3. Synthesis of 1 as a stereomeric mixture. TMS, trimethylsilyl; TMEDA, tetrametiletilendiamina.

( \pm )-3 were performed under pseudo-

high dilution conditions to avoid polymerization processes. Whereas $\mathrm{CuCl}$ and $\mathrm{CuCl}_{2}$ catalytic system is often employed for acetylene homocoupling, ${ }^{29}$ this strategy was not valid in this study, leading to a complete loss of the starting material with no sign of the desired product. As an alternative, the $\mathrm{Pd}\left(\mathrm{PPh}_{3}\right)_{2} \mathrm{Cl}_{2}$ and $\mathrm{Cul}$ catalytic system, one also employed for acetylenic homocoupling reactions, ${ }^{30}$ rendered the desired macrocycle 1 in $37 \%$ yield as a stereomeric mixture.

Examination of ${ }^{1} \mathrm{H}$ NMR spectrum after flash chromatography of the crude mixture uncovered the expected diastereoisomeric mixture of $(P, P, P)-\mathbf{1}$ and/or $(M, M, M)-\mathbf{1}$ and $(P, P, M)-\mathbf{1}$ and/or $(M, M, P)-1$ as ascertained by the number of non-equivalent protons (Figure S4). Given the fact that enantiomers are indistinguishable in standard NMR experiments, $(P, P, P)-\mathbf{1}$ and $(M, M, M)-\mathbf{1}$

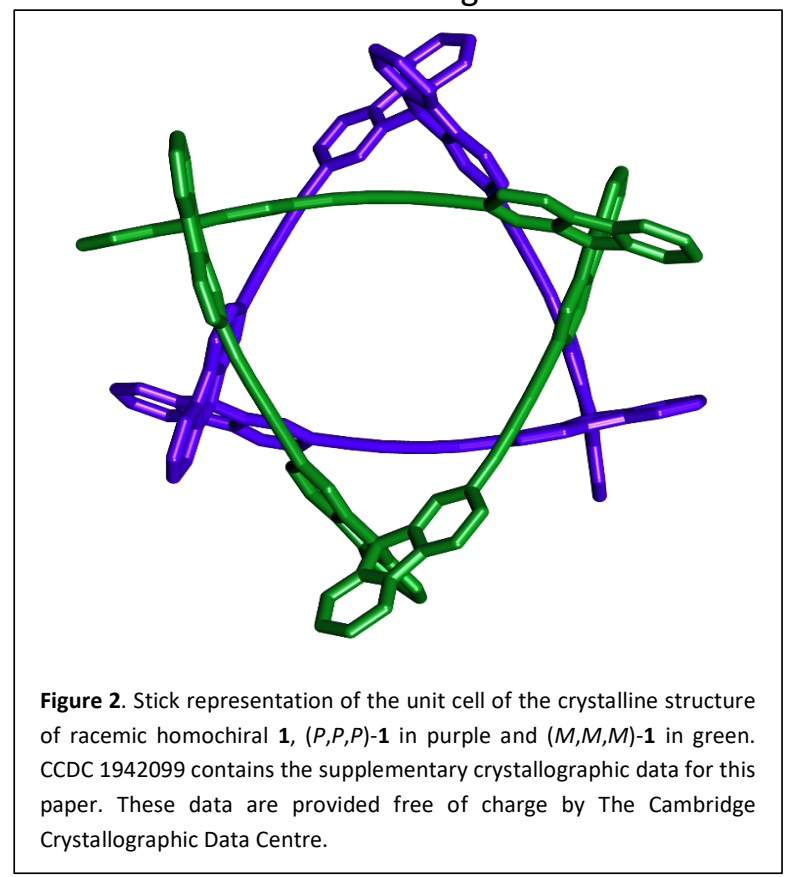

should have only seven non-equivalent protons with the same intensity for all. This stems from the presence of a $C_{3}$ and three $C_{2}$ symmetry axes making all fluorene units magnetically equivalent. On the other hand, for $(P, P, M)-\mathbf{1}$ and $(M, M, P)-\mathbf{1}$ featuring only a $C_{2}$ symmetry axis, the number of non-equivalent protons is 21 . Recrystallization of the stereomeric mixture from a solution of hexane $/ \mathrm{CH}_{2} \mathrm{Cl}_{2}$ gave rise to crystals of $(P, P, P)-1$ and $(M, M, M)-1$ while $(P, P, M)-\mathbf{1}$ and $(M, M, P)-\mathbf{1}$ remained in solution. After filtration, characterization by ${ }^{1} \mathrm{H}$ and ${ }^{13} \mathrm{C} N M R$ spectroscopy of the two fractions confirmed the predicted analyses discussed above (Figures S5-S8). 
Analysis of homochiral 1 by X-ray diffraction supported the structure obtained from the DFT calculations (Figure 2). The racemic crystallizes in the centrosymmetric $P$-1 presents one molecule of 1 with two $\mathrm{CH}_{2} \mathrm{Cl}_{2}$ solvent molecules in the asymmetric unit. While the symmetry is reduced compared with the simulations, the average absolute value of $\theta$ is $33^{\circ}$, very close to $30^{\circ}$, and therefore, fairly in the optimal range postulated above.

The absorption spectra of the two diastereomers of $\mathbf{1}$ are similar, exhibiting distinct peaks at 377 and 351 $\mathrm{nm}$ for $(P, P, P)-\mathbf{1} /(M, M, M)-\mathbf{1}$, and 380 and $354 \mathrm{~nm}$ for $(P, P, M)-\mathbf{1} /(M, M, P)-\mathbf{1}$. The complex spectra are due to the appearance of several electronic transitions and exciton and vibronic coupling as will be discussed below. Both compounds are good fluorescence emitters with emission in 390-450 nm range, quantum yields of ca. $30 \%$ and lifetimes of $0.31 \mathrm{~ns}$ for $(P, P, P)-\mathbf{1} /(M, M, M)-\mathbf{1}$, and $0.41 \mathrm{~ns}$ for $(P, P, M)-\mathbf{1} /(M, M, P)$-1 (Figures S17-S19). Remarkably, the thermogravimetric analysis unveiled high stability for each diastereomer up to $270{ }^{\circ} \mathrm{C}$ (Figures S21 and S22).

\section{Chiroptical responses}

Enantiomeric resolutions of $(P, P, P)-\mathbf{1} /(M, M, M)-\mathbf{1}$ and $(P, P, M)-\mathbf{1} /(M, M, P)-\mathbf{1}$ were performed through semipreparative HPLC using the chiral stationary phase Chiralpak ${ }^{\circledR}$ IA. Electronic circular dichroism (ECD) of each stereoisomer showed g-factor values up to 0.008 , and therefore the SBF systems herein are among organic systems presenting the strongest chiroptical responses (Figure 3 left). ${ }^{31}$
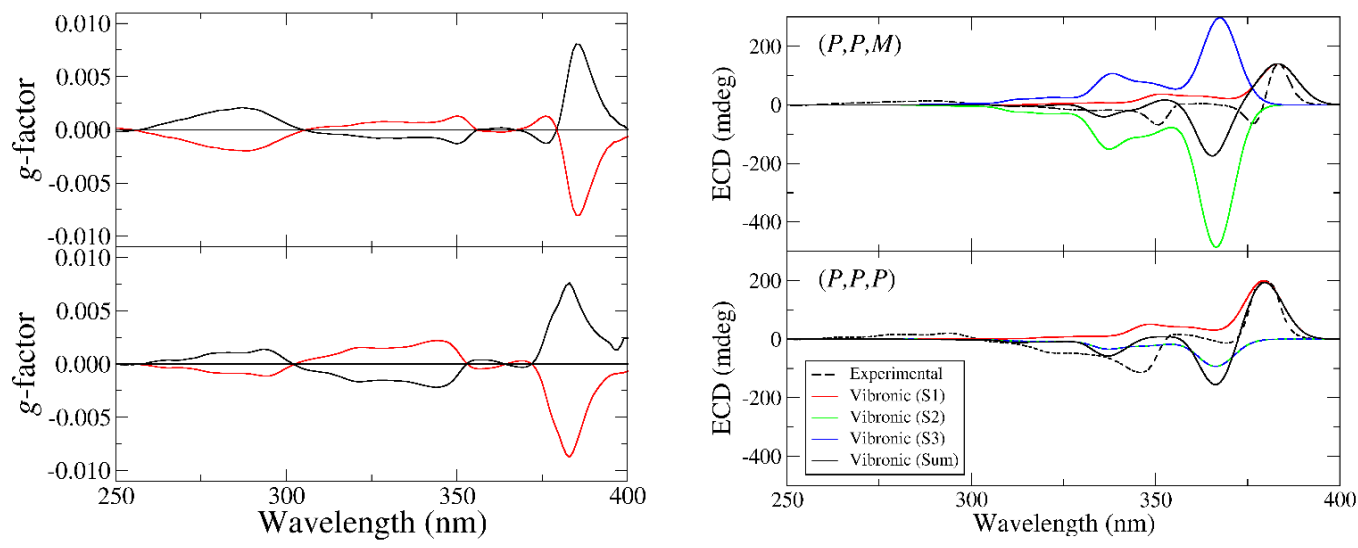

Figure 3. (left) $g$-Factor vs wavelength plot of the electronic circular dichroism spectra of heterochiral (top) and homochiral (bottom) 1 measured in acetonitrile. Fractions $A$ and $B$ from the enantiomeric resolution are shown in black and red, respectively. (right) ECD vibronic spectra for the three lowest states of $(P, P, M)-\mathbf{1}$ (top) and $(P, P, P)-\mathbf{1}$ (bottom) computed in Franck-Condon approximation with harmonic Vertical Gradient (VG) model $^{43}$ and convoluted with a Gaussian with $\mathrm{HWHM}=0.05 \mathrm{eV}$. The computed spectra for $(P, P, M)-\mathbf{1}$ and $(P, P, P)-\mathbf{1}$ were respectively scaled by arbitrary factors and redshifted by 0.18 and $0.23 \mathrm{eV}$, to superimpose the calculated results (Vibronic Sum) with the experiments.

In order to assign the absolute configuration (AC) of the different fractions from the enantiomeric resolution, we studied the absorption and ECD spectra of $(P, P, P)-\mathbf{1}$ and $(P, P, M)-\mathbf{1}$. Based on the fact that charge-transfer states likely exist in a large system made up of identical subunits, the long-range corrected CAM-B3LYP functional was employed for a more accurate prediction in combination with the 6-31G(d) basis set. The initial simulation of the absorption properties at a pure electronic level by simply convoluting the oscillator strength (OS) and rotatory strength (RS) with a Gaussian function did not provide a reliable similarity with the experimental spectra (Figure S23). The reason for such disagreement between experimental and predicted spectra could be due to a strong vibronic coupling. In fact, the energy difference between the two absorption experimental bands is about $2250 \mathrm{~cm}^{-1}(0.28 \mathrm{eV})$, which nicely matches with the typical triple bond stretching vibrational frequency. To analyse this possibility, we computed the vibronic spectra of S1, S2 and S3 at Franck-Condon level in harmonic approximation with our code FCclasses $3 .^{32}$ Results indicate that different bands observed in the spectrum of each electronic state arise from progressions along the CC triple bonds and their combined effect improves the agreement with experiment. The discrepancies found in band intensities could be attributed to an overestimation of the energy gap between S1 and S2/S3 and the existence of strong Jahn-Teller and pseudo-Jahn-Teller couplings ${ }^{33}$ among these three states, whose treatment is out of the scope of this paper. Nevertheless, the contribution of the individual states results in an overall agreement with the experiments for the two isomers investigated, allowing the confident AC assignment of different fractions from the enantiomeric resolution (Figure 3 right, for more details, see the 
$\mathrm{SI})$. Thus, fraction $\mathrm{A}$ of homochiral 1 has $(P, P, P)$ AC and fraction $\mathrm{A}$ of heterochiral 1 has $(P, P, M)$ AC. Consequently, fraction $B$ of homochiral 1 has $(M, M, M) A C$ and fraction $B$ of heterochiral 1 has $(M, M, P) A C$.

\section{Helical molecular orbitals}

Molecular orbitals of $(P, P, P)-\mathbf{1}$ and $(P, P, M)-\mathbf{1}$ were evaluated at the B3LYP/6-31G(d,p) level of theory in search of those with helical character (Figure 4). Whereas HOMO-5 for $(P, P, P)-1$ is of clear helical character in the three DPBD moieties with $\theta=30^{\circ}$, HOMO-5 of $(P, P, M)-1$ displays helicity in the two DPBDs with $\theta=15^{\circ}$ and no helical character is attributed to the DPBD with $\theta$ $=5^{\circ}$. These findings follow our theoretical model illustrated above.
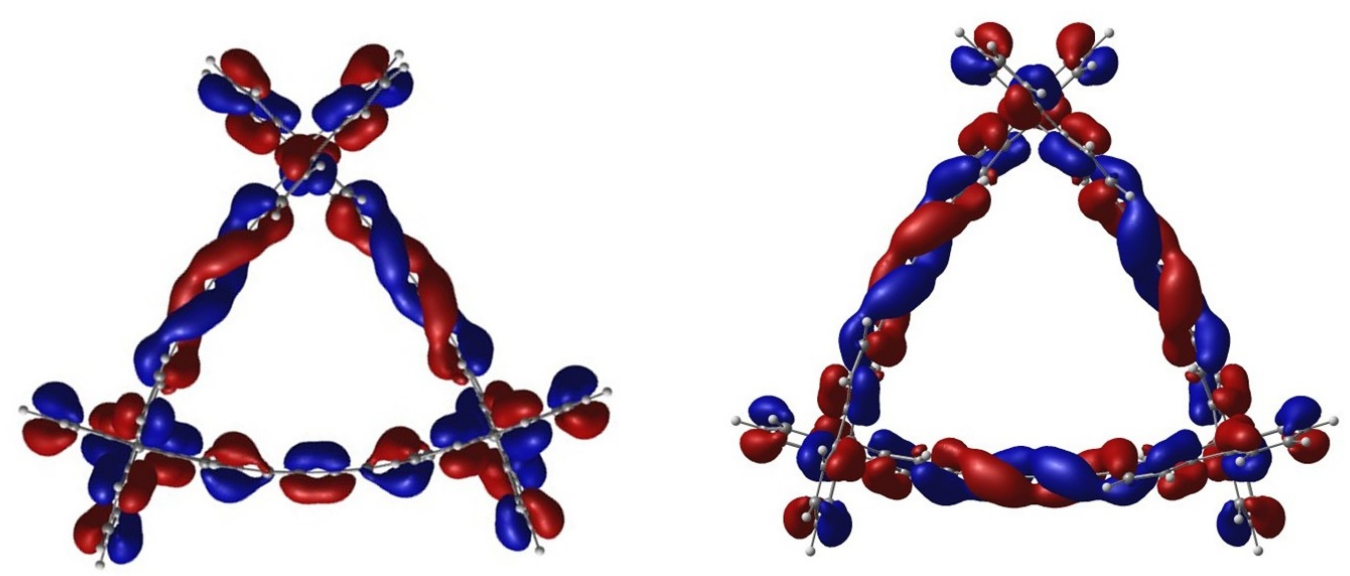

Figure 4. Representation of HOMO-5 for $(P, P, M)-\mathbf{1}$ (left) and $(P, P, P)-\mathbf{1}$ (right) at the B3LYP/6-31G(d,p) level of theory as implemented in Gaussian 09. Kohn-Sham orbitals shown with isosurface value $=0.015$.

The simultaneous presence of two and three DPBD moieties featuring Hel-MOs in heterochiral and homochiral 1, respectively, may have a dramatic impact on the responses of such systems.

\section{Interchromophore effects}

The common quaternary carbon atom in SBFs isolates the two fluorene constituent units. Apart from spiroconjugation, ${ }^{34-36}$ two electronically isolated chromophores can be considered in SBFs. Based on this principle, $(P, P, P)-\mathbf{1}$ would present three independent chromophores separated by the spiranic quaternary carbons (Figure 5). In order to evaluate how realistic is this assumption, we applied a pure exciton model ${ }^{36}$ for the prediction of the low energy part of the ECD spectrum of $(P, P, P)-\mathbf{1}$. To this end, we first computed the excited states of a single chromophore, and based on the analysis of the lowest excited states, considered only the S1 state to apply the exciton model. Couplings between the three S1 monomeric excitations were computed as a Coulombic interaction of their transition densities with

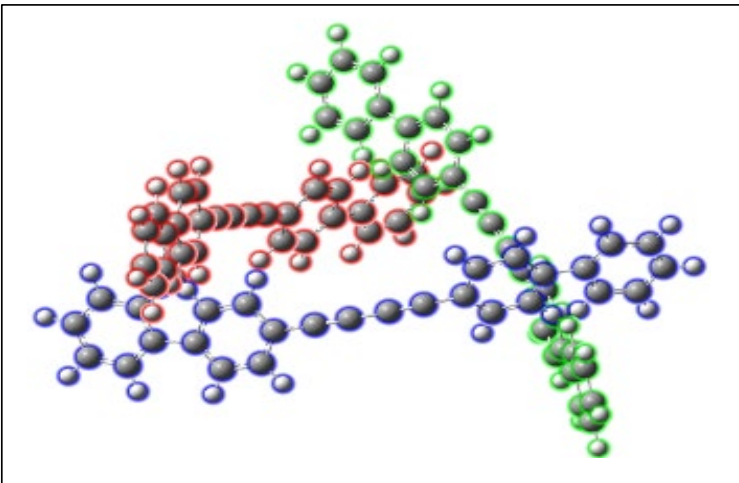

Figure 5. Model of $(P, P, P)-1$ represented by three independent chromophores in red, blue and green. For technical reasons, the quaternary carbons were removed and the valences saturated with hydrogens.

Gaussian 16 and ECD spectra were computed with EXAT code. ${ }^{37-40}$ The obtained results are in very good agreement with those predicted by TD-DFT, strongly supporting the thesis that the strong ECD is due to exciton couplings of local excitations on the butadiyne moieties following the selection rules imposed by symmetry (Figure S26, for more details, see the SI). ${ }^{41,42}$ Therefore, we conclude that the DPBD moieties present in these SBF macrocycles interact through an exciton coupling mechanism, at least for low excited 
states. However, since the Hel-MOs featured by $\mathbf{1}$ are separated from the energy of the HOMO, their contribution is only significant for highly excited states which are located in an energy region where the density of states is high, and contributions, other than exciton coupling, may have a significant impact.

\section{Conclusions}

Helical molecular orbitals are receiving increasing attention by the scientific community. Nevertheless, the presence of degenerated or pseudo-degenerated orbitals featuring opposite helicities is probably the main obstacle to experimentally explore the scope of molecular orbital helicity. To tackle this challenge, we have achieved distinct helical molecular orbitals by controlling the torsion angle between the terminal groups in diphenylbutadiyne fragments. On the basis of this hypothesis, we designed and synthesised all possible stereoisomers of cyclic diethynylspirobifluorene 1. NMR spectroscopy and X-ray diffraction analysis have confirmed the postulated geometries. Additionally, the comparison of experimental with predicted electronic circular dichroism enabled the absolute configuration assignment of all stereoisomers. Analysis of the molecular orbitals of 1 verified our strategy to access systems featuring distinct helical molecular orbitals. Finally, a good agreement between TD-DFT and pure exciton model for the prediction of the main features of the ECD spectrum let us foresee the synergetic effects on responses where MOs featuring helicities in more than one DPBD fragments are involved. Notably, the developed systems present high stability and remarkable photophysical and chiroptical responses. Thus, the present study opens the possibility to explore the scope of real systems presenting helical frontier molecular orbitals, a goal we are currently pursuing.

\section{Conflicts of interest}

The are no conflicts to declare.

\section{Acknowledgements}

This work was funded by Xunta de Galicia (ED431F 2016/005 and GRC2019/24), IIS Galicia Sur, and IBEROS (0245_IBEROS_1_E). A.O. thanks Xunta de Galicia for predoctoral fellowship. J.L.A.-G. thanks the Spanish Ministerio de Economía y Competividad (MINECO) for a "Ramón y Cajal" research contract. All authors gratefully acknowledge CACTI de Vigo. The authors also would like to thank A. Acuña Couña (CACTI de Vigo) for his support in the enantiomeric resolution and TGA analysis and L. M. Salonen (INL Portugal) for her valuable discussions. D.A. thanks Fundación Ramón Areces for fundings, University of Malaga Servicio de Supercomputación y Bioinformática for computational resources, and Pisa Unit of ICCOM-CNR for hospitality. The work in Madrid was supported by the MINECO-FEDER project CTQ2017-87054, by the "Severo Ochoa" program for Centers of Excellence in R\&D of the MINECO (SEV-2016-0686) and by the Campus of International Excellence (CEI) UAM+CSIC. L.W. acknowledges an Erasmust grant of the European Commission.

\section{Notes and references}

1 F. Diederich and M. Kivala, Adv. Mater., 2010, 22, 803-812.

2 A. Hoffmann-Röder and N. Krause, Angew. Chemie Int. Ed., 2004, 43, 1196-1216.

3 J. A. Januszewski and R. R. Tykwinski, Chem. Soc. Rev., 2014, 43, 3184-3203.

4 P. Rivera-Fuentes and F. Diederich, Angew. Chem. Int. Ed., 2012, 51, 2818-28.

5 C. H. Hendon, D. Tiana, A. T. Murray, D. R. Carbery and A. Walsh, Chem. Sci., 2013, 4, 4278-4284.

6 Y. Orimoto, Y. Aoki and A. Imamura, J. Phys. Chem. C, 2019, 123, 11134-11139.

7 A. Imamura and Y. Aoki, Chem. Phys. Lett., 2013, 590, 136-140.

8 M. D. Peeks, P. Neuhaus and H. L. Anderson, Phys. Chem. Chem. Phys., 2016, 18, 52645274. 

16146. 4598-4608.

R. Naaman, Y. Paltiel and D. H. Waldeck, Nat. Rev. Chem., 2019, 3, 250-260. Striccoli, E. Fanizza, R. Naaman, D. H. Waldeck, D. Porath and Y. Paltiel, Small, 2019, 15, e1804557.

O. Ben Dor, S. Yochelis, S. P. Mathew, R. Naaman and Y. Paltiel, Nat. Commun., 2013, 4, $1-6$.

A. Kumar, E. Capua, C. Fontanesi, R. Carmieli and R. Naaman, ACS Nano, 2018, 12, 3892-3897.

16 W. Mtangi, F. Tassinari, K. Vankayala, A. Vargas Jentzsch, B. Adelizzi, A. R. A. Palmans, C. Fontanesi, E. W. Meijer and R. Naaman, J. Am. Chem. Soc., 2017, 139, 2794-2798.

17 N. Harada and K. Nakanishi, Acc. Chem. Res., 1972, 5, 257-263.

182013 M. J. Frisch et al. Gaussian 09, Revision D.01 Gaussian, Inc., Wallingford CT, 2013.

19 M. H. Garner, R. Hoffmann, S. Rettrup and G. C. Solomon, ACS Cent. Sci., 2018, 4, 688700.

20 S. Odermatt, J. L. Alonso-Gómez, P. Seiler, M. M. Cid and F. Diederich, Angew. Chem. Int. Ed., 2005, 44, 5074-5078.

21 S. Míguez-Lago, A. L. Llamas-Saiz, M. M. Cid and J. L. Alonso-Gómez, Chem. A Eur. J., 2015, 21, 18085-18088.

22 A. Ozcelik, R. Pereira-Cameselle, A. Von Weber, M. Paszkiewicz, M. Carlotti, T. Paintner, L. Zhang, T. Lin, Y.-Q. Zhang, J. V. V. Barth, T. van den Nobelen, R. C. C. Chiechi, M. Jakob, U. Heiz, S. Chiussi, A. Kartouzian, F. Klappenberger and J. L. Alonso-Gómez, Langmuir, 2018, 34, 4548-4553.

23 J. L. Alonso-Gómez, P. Schanen, P. Rivera-Fuentes, P. Seiler and F. Diederich, Chem. Eur. J., 2008, 14, 10564-10568.

24 C. Poriel, J. Rault-Berthelot and L. J. Sicard, Chem. Commun., 2019, 55, 14238-14254.

25 S. Castro-Fernández, M. M. Cid, C. S. López and J. L. Alonso-Gómez, J. Phys. Chem. A, 2015, 119, 1747-1753.

26 A. Ozcelik, Á. Peña-Gallego, R. Pereira-Cameselle and J. L. Alonso-Gómez, Chirality, 2020, 32, 464-473.

27 V. C. Arias-Coronado, R. Pereira-Cameselle, A. Ozcelik, M. Talavera, Á. Peña-Gallego, J. L. Alonso-Gómez and S. Bolaño, Chem. A Eur. J., 2019, 22, 13496-13499.

28 S. Castro-Fernández, R. Yang, A. P. García, I. L. Garzón, H. Xu, A. G. Petrovic and J. L. Alonso-Gómez, Chem. A Eur. J., 2017, 23, 11747-11751.

29 I. R. Lahoz, A. Navarro-Vázquez, A. L. Llamas-Saiz, J. L. Alonso-Gómez and M. M. Cid, Chem. Eur. J., 2012, 18, 13836-13843. 
I. R. Lahoz, A. Navarro-Vázquez, J. L. Alonso-Gómez and M. M. Cid, Eur. J. Org. Chem., 2014, 2014, 1915-1924.

31 S. Míguez-Lago, M. M. Cid and J. L. Alonso-Gómez, Eur. J. Org. Chem., 2016, 2016, $5716-5721$.

32 available upon request F.Santoro, FCclasses 2.1, freely available at http://www.pi.iccom.cnr.it/fcclasses, alast accessed March 2020. F. Santoro, J. Cerezo, FCclasses 3, 2019, .

33 S. Mahapatra, L. S. Cederbaum and H. Ko, Chem. Phys., 1999, 111, 10452-10463.

34 H. E. Simmons and T. Fukunaga, J. Am. Chem. Soc., 1967, 89, 5208-5215.

35 D. Padula, D. Picconi, A. Lami, G. Pescitelli and F. Santoro, J. Phys. Chem. A, 2013, 117, 3355-3368.

36 D. Padula, F. Santoro and G. Pescitelli, RSC Adv., 2016, 6, 37938-37943.

37 J. R. M. J. Frisch, G. W. Trucks, H. B. Schlegel, G. E. Scuseria, M. A. Robb and I. Cheeseman, et al., Gaussian, 2016.

38 S. Jurinovich, L. Cupellini, C. A. Guido and B. Mennucci, J. Comput. Chem., 2018, 39, 279-286.

39 S. Jurinovich, G. Pescitelli, L. Di Bari and B. Mennucci, Phys. Chem. Chem. Phys., 2014, 16, 16407-16418.

40 S. Jurinovich, C. A. Guido, T. Bruhn, G. Pescitelli and B. Mennucci, Chem. Commun., 2015, 51, 10498-10501.

41 S. Castro-Fernández, Á. Peña-Gallego, R. A. Mosquera and J. L. Alonso-Gómez, Molecules, 2019, 24, 141.

42 A. Ozcelik, R. Pereira-Cameselle, R. A. Mosquera, Á. Peña-Gallego and J. L. AlonsoGómez, Symmetry 2019, Vol. 11, Page 1245, 2019, 11, 1245.

43 F. J. Avila Ferrer and F. Santoro, Phys. Chem. Chem. Phys., 2012, 14, 13549-13563. 